\title{
Studies towards enzymatic kinetic resolutions of 1,3-diol peptidomimetics obtained via the Ugi reaction
}

\author{
Szymon Kłossowski, Adam Redzej, Sara Szymkuć, and Ryszard Ostaszewski* \\ Institute Of Organic Chemistry PAS, Kasprzaka 44/52, 01-224 Warsaw \\ E-mail: ryszard.ostaszewski@icho.edu.pl
}

This paper is dedicated to Prof. Janusz Lipkowski on the occasion of his 70th birthday

\begin{abstract}
Enzymatic methods in combination with the multicomponent Ugi condensation make a very efficient method for simple synthesis of non-racemic peptidomimetics. The aim of the studies was to develop an enzymatic kinetic resolution of 1,3-diol peptidomimetics providing nonracemic compounds. Among many applications, 1,3-diols can serve as intermediates in the synthesis of anticancer agents - $\beta$-acyloxymethacrylic amides. Stereoselective enzymatic acylation and hydrololysis of Ugi products were investigated. The enantiomeric or diastereomeric excesses were determined in both cases. As a result, an efficient enzymatic method for the synthesis chiral, non-racemic 1,3-diol peptidomimetics was developed.
\end{abstract}

Keywords: Kinetic resolution, enzymatic hydrolysis, peptidomimetics

\section{Introduction}

Enzymes have become common catalysts for kinetic resolution of racemic mixtures. Numerous examples of chiral, non-racemic compounds have been obtained via enzymatic hydrolysis or acylation. ${ }^{1-3}$ Interesting combinations of enzymatic procedures and multicomponent reactions, such as the Passerini ${ }^{4}$ or Ugi reactions, ${ }^{5-6}$ have been reported. Isocyanide-based, multicomponent reactions are very useful tools in chemistry and combinatorial chemistry. ${ }^{7-10}$ The Ugi reaction is a four-component condensation of an aldehyde, amine, carboxylic acid and an isocyanide that provide $\alpha$-acylaminoamides. ${ }^{7}$ Although recently, the assymetric, catalytic versions of Ugi threecomponent reactions were described, ${ }^{11-13}$ the stereocontrol of the classical, four-component Ugi reaction still remains very limited. We reported previously that enzymatic desymmetrization of cyclic anhydrides and subsequent Ugi reaction can be used for the synthesis of non-racemic small peptide analogues in a one-pot procedure. ${ }^{5}$ Another example of the combination of the 
biocatalytic method and the Ugi reaction is the enzymatic oxidation of a substituted pyrrolidine to an optically active 1-pyrroline, which was subsequently used in an asymmetric Ugi reaction. ${ }^{6}$

In this paper we present our studies on the enzymatic desymmetrization of 1,3-diol peptidomimetics I obtained via the Ugi reaction (

Figure 1). Similar 1,3-diol fragments were previously found in anticonvulsant agents, kinase inhibitors as well as an achiral alternative for 1,2-diols in asthma therapy. ${ }^{14-16} \mathrm{We}$ also have recently described 1,3-diol I as an intermediate for the synthesis of peptidomimetics possessing various electrophilic fragments including a new class of $\beta$-acyloxymethacrylates - potent anticancer agents. ${ }^{17}$ Peptidomimetics I were previously obtained via the classic Ugi reaction as a racemic mixture. The proper method of the synthesis non-racemic diol I can be applied for further synthesis of optically active, anticancer $\beta$-acyloxymethacrylates.

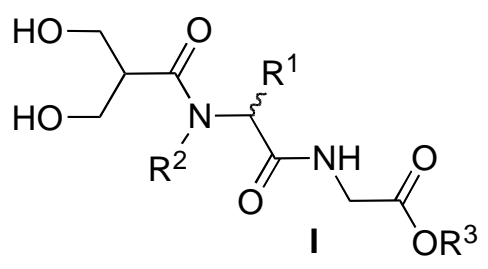

1: $\mathrm{R}^{1}=i-\mathrm{Bu} ; \mathrm{R}^{2}=\mathrm{Bn} ; \mathrm{R}^{3}=\mathrm{Bn}$

2: $\mathrm{R}^{1}=i-\mathrm{Bu} ; \mathrm{R}^{2}=\mathrm{DMB} ; \mathrm{R}^{3}=\mathrm{Et}$

Figure 1. General structure I of 1,3-diol peptidomimetics.

\section{Results and Discussion}

The synthetic strategies, presented in Scheme 1, are based on the enzymatic kinetic resolution of enantiomers of compounds $\mathbf{I}$. Considering the structure of $\mathbf{I}$, two approaches have been proposed. The first method is based on enzymatic acylation of compound $\mathbf{1}$ to alcohol $\mathbf{4}$. The second approach is based on the enzymatic hydrolysis reaction of the ester group to an acid 5. For the first approach, diol $\mathbf{1}$ as its benzyl ester was chosen, since it was judged to be less likely to undergo side reactions involving the ester moiety, than an ethyl ester. For the enzymatic hydrolysis, diol $\mathbf{2}$ was chosen (

Scheme 1).
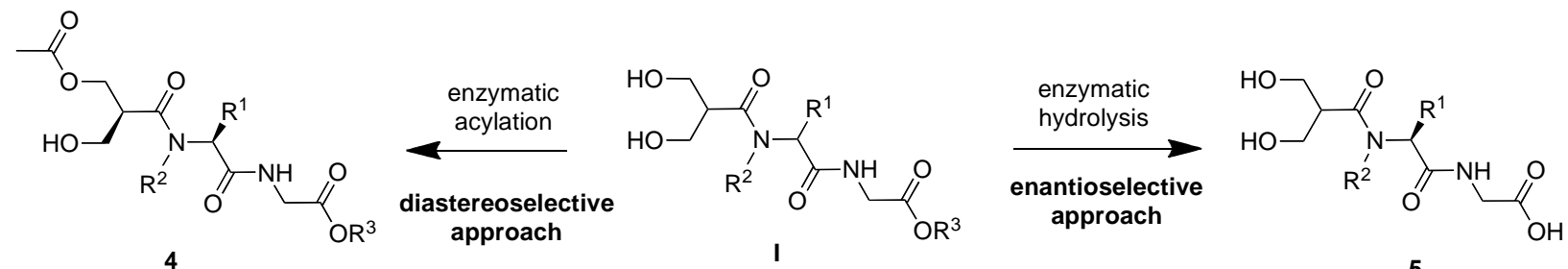
Scheme 1. Synthetic strategy for kinetic resolution of 1,3 diol peptidomimetics $\mathbf{I}$.

The synthesis of substrates for enzymatic reaction. The synthesis of diol $\mathbf{1}$ was described previously. ${ }^{17}$ For the synthesis of analogue 2, similar conditions were applied (Scheme 2). Compound 7 was obtained in the Ugi reaction with acid 3, ethyl isocyanoacetate, 2,4dimethoxybenzylamine and isovaleric aldehyde in $49 \%$ yield (Scheme 2). Then the acetal protecting group was cleaved in $80 \%$ aq acetic acid providing diol 2 in $77 \%$ yield.
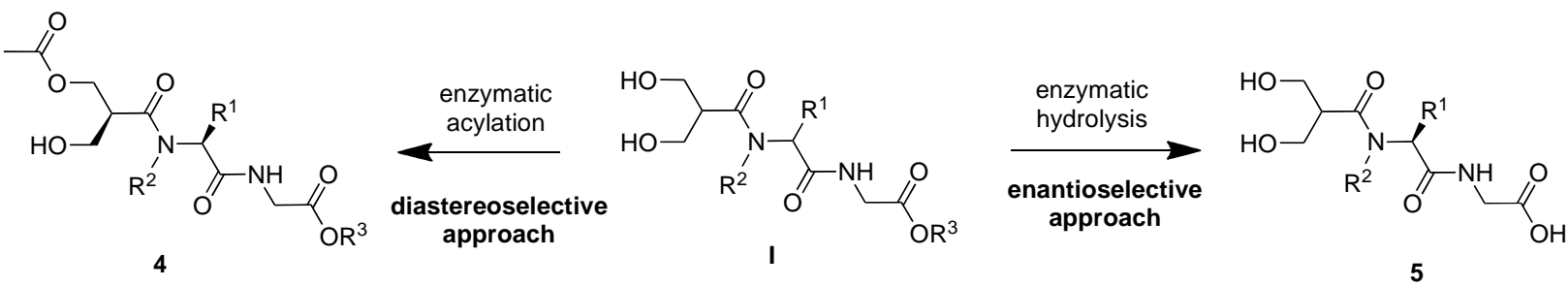

Scheme 2. The Synthesis of the substrates for lipases. Reagents and conditions: (a) EtOH, $24 \mathrm{~h}$, rt, $49 \%$; (b) $80 \%$ aq $\mathrm{CH}_{3} \mathrm{COOH}$, overnight, rt, $77 \%$.

Diasteroselective approach. Since compound 1 contains two diastereotopic hydroxyl groups, the monoacylation reaction can lead to a mixture of stereoisomers. As can be seen in Scheme 3, the acylation reaction of $\mathbf{1}$ should be catalyzed diastereoselectively and enantioselectively, in order to provide efficient desymmetrisation. Initially, the diastereoselectivity of enzymatic acylation with vinyl acetate was studied as described before. ${ }^{18}$<smiles>CC(=O)OC[C@H](CO)C(=O)N(Cc1ccccc1)[C@H](CC(C)C)C(=O)NCC(=O)OC[C@H](CO)C(=O)N(Cc1ccccc1)[C@H](CC(C)C)C(=O)NCC(=O)OCc1ccccc1</smiles>

a)<smiles>CC(=O)OC[C@H](CO)C(=O)N(Cc1ccccc1)[C@@H](CC(C)C)C(=O)NCC(=O)OCc1ccccc1</smiles>

Scheme 3. Enzymatic acylation of 1,3 diol 1. Reagents and conditions: a) enzyme, vinyl acetate, DIPE, $40{ }^{\circ} \mathrm{C}, 2-7$ days.

In our previous studies, it was found that the proper choice of solvent is crucial for stereoselectivity of an enzyme. ${ }^{19}$ Thus different solvents were examined, such as: toluene, vinyl 
acetate, 1,4-dioxane, tert-butyl methyl ether (TBME). After enzymatic screening of different reaction conditions, seven enzymes were found to catalyze the reaction. (For the full list of enzymes tested, see the Experimental Section, 4.7) The results are presented in Table 1.

Table 1. Initial screening of acylation reaction of $\mathbf{1}$

\begin{tabular}{|c|c|c|c|}
\hline Entry & Solvent & Enzyme & d.e. ${ }^{\mathrm{a}}[\%]$ \\
\hline 1 & \multirow{7}{*}{$i-\mathrm{Pr}_{2} \mathrm{O}$} & Novozym 435 & 3 \\
\hline 2 & & Pseudomonas fluorescens & 11 \\
\hline 3 & & Pseudomonas cepaciae & 32 \\
\hline 4 & & Aspergillus oryzae & 48 \\
\hline 5 & & Mucor javanicus & 16 \\
\hline 6 & & Pennicilium roqueforti & 11 \\
\hline 7 & & Candida antarctica & 9 \\
\hline 8 & \multirow{3}{*}{ TBME } & Novozym 435 & 9 \\
\hline 9 & & Pseudomonas fluorescens & 12 \\
\hline 10 & & Pseudomonas cepaciae & 20 \\
\hline
\end{tabular}

${ }^{a}$ Determined by RP-HPLC (Method A)

As summarized in the Table 1, diol $\mathbf{1}$ can be acylated in a chemoselective way (only monoacylation product was observed in the reaction mixture). The Novozym 435 and the lipases from: Pseudomonas fluorescens, Pseudomonas cepaciae, Aspergillus oryzae, Mucor javanicus, Penniclium roqueforti and Candida antarctica were able to catalyze the reaction. The highest diastereoselectivity was observed when lipase from Aspergillus oryzae was used (entry 4). These results were still not satisfactory, therefore the enantioselectivity was not further studied.

Enantioselective approach. Since the enzymatic acylation of diol 1 was not very selective, the second approach was based on enzymatic enantioselective hydrolysis. In this approach the number of possible products is reduced, which simplifies stereochemical course of the reaction. Formation of only the carboxylic acid $\mathbf{5}$ and unreacted substrate $\mathbf{2}$ is observed.<smiles>CCOC(=O)CNC(=O)C(CC(C)C)N(Cc1cc(OC)ccc1OC)C(=O)C(CO)CO</smiles>

(rac)-2

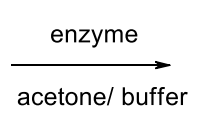

acetone/ buffer

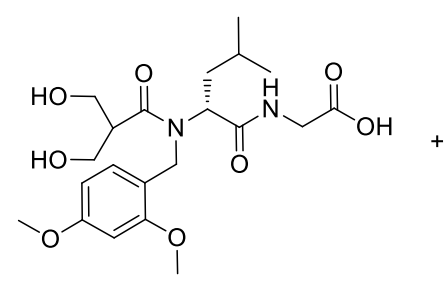

5

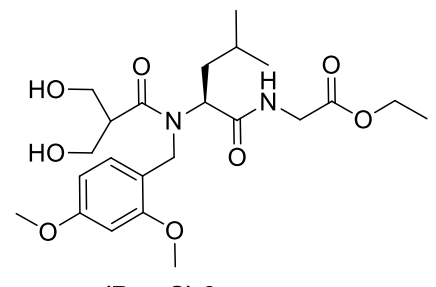

( $R$ or $S)-2$

Scheme 4. Enzymatic hydrolysis of 1,3-diol 2. Reagents and conditions: see Table 2. 
For the initial screening, the typical conditions were applied. In order to increase solubility of substrate, acetone was used as a co-solvent. Among all the tested enzymes, six of them catalyzed the reaction. The results are summarized in Table 2.

Table 2. Enzymatic hydrolysis of 2

\begin{tabular}{|c|c|c|c|c|c|}
\hline Entry & Enzyme & Time $[\mathrm{d}]$ & Conv.[\%] ${ }^{\mathrm{a}}$ & $\mathrm{ee}_{\mathrm{s}}[\%]^{\mathrm{b}}$ & $E^{\mathrm{c}}$ \\
\hline 1 & PLAP & 28 & 40 & 18 & 2.1 \\
\hline 2 & Porcine pancreas lipase type II & 4 & 27 & 15 & 2.7 \\
\hline 3 & Papaine & 6 & 35 & 28 & 4.1 \\
\hline 4 & $\begin{array}{l}\text { Macerase Pektynase from } \\
\text { Rhizopus sp. }\end{array}$ & 2 & 26 & 26 & 8.6 \\
\hline 5 & Candida antarctica lipase & 2 & 53 & $>99.9$ & 120 \\
\hline 6 & Rhizopus arrhizus lipase & 2 & 40 & 82 & 21 \\
\hline
\end{tabular}

${ }^{a}$ Yields determined by RP-HPLC (Method C). ${ }^{b} e_{s}$ determined by HPLC (Method B). ${ }^{\mathrm{c}}$ Enantioselectivity $(E)$ was calculated according to equation:

$$
E=\frac{\ln \left[(1-c)\left(1-e e_{s}\right)\right]}{\ln \left[(1-c)\left(1+e e_{s}\right)\right]}
$$

The best results were obtained when porcine lipase acetone powder, porcine pancreas lipase type II, papaine, pektynase, CALB and lipase from Rhisopus arrhisus were used as biocatalysts. The enatiomeric excess of the substrate was determined by HPLC. The acetone powder required a very long reaction time and despite moderate conversion, the enantioselectivity was very poor. Interestingly, papain exhibited lipase activity and catalyzed the reaction. The best results were obtained in the case of Rhisopus arrhisus lipase and Candida antarctica lipase which catalyzed the reaction with excellent enantioselectivity (Table 2, entries 5 and 6).

\section{Conclusions}

Herein we have described the results of our studies on enzymatic kinetic resolution of 1,3-diol peptidomimetics using two different approaches. The enzymatic diasteroselective acylation of hydroxyl groups was not efficient and therefore not optimized. On the other hand, the enantioselective enzymatic hydrolysis of the ester group was found to be very efficient and the respective carboxylic acid $\mathbf{5}$ was obtained with excellent enantioselectivity. The proposed methodology is simple, efficient and could be expanded to other type of substrates. In future studies this methodology will be applied for the synthesis of non-racemic $\beta$-acyloxyamides. 


\section{Experimental Section}

General. NMR spectra were measured with Varian $200 \mathrm{MHz}$ and Varian $400 \mathrm{MHz}$ spectrometers, with TMS used as an internal standard. Thin layer chormatography was performed with silica gel 60 (230-400 mesh or 70-230 mesh, Merck) and silica gel 60 (Merck). CHN analysis was performed on a Vario EL III (Elementor) elemental analyzer. High-resolution mass spectrometry (HR-MS) spectra were recorded on a Mariner (PerSeptive Biosystems) apparatus. HPLC experiments were carried out on a Shimadzu or Varian apparatus, Method A: Kromasil C-18 column, 250 x $4.0 \mathrm{~mm} ; \lambda=250 \mathrm{~nm}$; eluent: MeOH/water 4:1 (v/v), flow rate: 1 $\mathrm{mL} / \mathrm{min}$; method B: CHIRACEL OD-H column), $\lambda=232 \mathrm{~nm}$ eluent: hexane/isopropanol 9:1 (v/v), flow rate: $1 \mathrm{~mL} / \mathrm{min}$; method C: column: Kromasil C-18 column, 250 x $4.0 \mathrm{~mm} ; \lambda=231$ nm; eluent: $\mathrm{MeOH} /$ water 7:3 (v/v), flow rate: $1 \mathrm{~mL} / \mathrm{min}$.

\section{\{2-[Benzyl-(1,5-dioxaspiro[5.5] undecane-3-carbonyl)amino]-4-methylpentanoylamino\}} acetic acid benzyl ester (6). To a solution of isovaleric aldehyde $(0.25 \mathrm{~mL}, 2.3 \mathrm{mmol})$ in ethanol $(2 \mathrm{~mL})$ benzylamine was added $(0.25 \mathrm{~mL}, 2.3 \mathrm{mmol})$. The mixture was stirred for $30 \mathrm{~min}$ and 1,5-dioxospiro[5.5] undecancarboxylic acid (3, $457 \mathrm{mg}, 2.3 \mathrm{mmol})$ was added. The reaction mixture was cooled to $0{ }^{\circ} \mathrm{C}$ and benzyl isocyanoacetate was added (400 mg, $2.3 \mathrm{mmol}$ ) then it was stirred for $24 \mathrm{~h}$ at room temperature. Ethanol was evaporated and the residue was purified by flash chromatography (silica gel, $\mathrm{R}_{f} 0.16$, hexane:ethyl acetate, 8:2, v:v) giving a colorless oil (614 mg, 48\%). ${ }^{1} \mathrm{H}$ NMR (200 MHz, $\left.\mathrm{CDCl}_{3}\right): \delta 0.80\left(\mathrm{~d}, J 6.8 \mathrm{~Hz}, 6 \mathrm{H}, \mathrm{C}\left(\mathrm{CH}_{3}\right)_{2}\right), 1.25-1.58(\mathrm{~m}$, $\left.12 \mathrm{H}, 6 \times \mathrm{CH}_{2}\right), 1.64-2.05\left(\mathrm{~m}, 1 \mathrm{H}, \mathrm{CH}\left(\mathrm{CH}_{3}\right)_{2}\right), 3.02-3.18\left(\mathrm{~m}, 1 \mathrm{H}, \mathrm{CH}_{2} \mathrm{CO}\right), 3.72-3.92(\mathrm{~m}, 4 \mathrm{H}$, $\left.\mathrm{OCH}_{2}\right), 4.00-4.34\left(\mathrm{~m}, 4 \mathrm{H}, \mathrm{CH}_{2} \mathrm{~N}\right), 4.61(\mathrm{~m}, 1 \mathrm{H}, \mathrm{NCHCO}), 5.16\left(\mathrm{~s}, 2 \mathrm{H}, \mathrm{ArCH}_{2}\right), 7.10-7.17(\mathrm{~m}$, $2 \mathrm{H}, \mathrm{ArH}), 7.23-7.38(\mathrm{~m}, 8 \mathrm{H}, \mathrm{ArH}) .{ }^{13} \mathrm{C} \mathrm{NMR}\left(50 \mathrm{MHz}, \mathrm{CDCl}_{3}\right): \delta 22.6,22.7,22.8,23.1,25.5$, 26.0, 27.7, 36.9, 38.3, 40.4, 41.4, 48.5, 55.9, 61.3, 61.6, 67.5, 98.4, 126.1, 127.9, 128.8, 128.9, $129.0,129.2,135.5,137.4,169.7,171.3,173.8$.

\{2-[Benzyl-(3-hydroxy-2-hydroxymethyl-propionyl)amino]-4-methylpentanoylamino\}acetic acid benzyl ester. (1) Compound $6(579 \mathrm{mg}, 1.0 \mathrm{mmol})$ was dissolved in acetic acid solution $(80 \% \mathrm{aq}, 10 \mathrm{~mL})$. The mixture was stirred at room temperature for $18 \mathrm{~h}$ then solvent was evaporated and the residue was purified by flash chromatography (silica gel, Rf 0.08, 5:5, hexane:ethyl acetate, v/v) to give the product as a colorless oil (353 mg, 71\%). ${ }^{1} \mathrm{H}$ NMR (200 $\mathrm{MHz}_{\mathrm{CDCl}}$ ): $\delta 0.72\left(\mathrm{~d}, J 6.4 \mathrm{~Hz}, 3 \mathrm{H}, \mathrm{CHCH}_{3}\right), 0.84\left(\mathrm{~d}, J 6.6 \mathrm{~Hz}, 3 \mathrm{H}, \mathrm{CHCH}_{3}\right), 1,40-1,58(\mathrm{~m}$, $\left.2 \mathrm{H}, \mathrm{CH}_{2} \mathrm{CH}\left(\mathrm{CH}_{3}\right)_{2}\right), 1.82-1.98\left(\mathrm{~m}, 1 \mathrm{H}, \mathrm{CH}\left(\mathrm{CH}_{3}\right)_{2}\right), 3.02-3.18(\mathrm{~m}, 1 \mathrm{H}, \mathrm{CHCO}), 3.25-3.53(\mathrm{~m}, 2 \mathrm{H}$, $\mathrm{OH}), 3.70-3.88$ (m, 4H, $\left.\mathrm{CH}_{2} \mathrm{OH}\right), 4.25-4.60$ (m, 2H, $\left.\mathrm{NCH}_{2} \mathrm{CO}\right), 5.00-5.22$ (m, 3H, $\left.\mathrm{ArCH}_{2}, \mathrm{NH}\right)$, 7.18-7.41 (m, 10H, ArH). ${ }^{13} \mathrm{C}$ NMR (50 MHz, $\left.\mathrm{CDCl}_{3}\right): \delta 14.5,21.3,22.5,23.0,25.6,36.7,41.4$, 46.8, 48.6, 56.4, 60.7, 62.3, 63.4, 67.8, 126.2, 127.7, 128.4, 128.7,129.0, 129.1, 135.2, 137.7, 171.0, 171.4, 178.3 Anal. calcd for $\mathrm{C}_{26} \mathrm{H}_{34} \mathrm{~N}_{2} \mathrm{O}_{6} \mathrm{X} 1 \mathrm{H}_{2} \mathrm{O}: \mathrm{C}, 63.92 ; \mathrm{H}, 7.43 ; \mathrm{N}, 5.73$; found: C, $63.92 ; \mathrm{H}, 7.27 ; \mathrm{N}, 5.62$.

\{2-[(2,4-Dimethoxybenzyl)-(1,5-dioxaspiro[5.5] undecane-3-carbonyl)amino]-4-ethyl-pentanoylamino\}acetic acid ethyl ester (7). To a solution of isovaleric aldehyde (160 $\mu \mathrm{L}, 1.5 \mathrm{mmol})$ 
in anhydrous ethanol $(2 \mathrm{~mL}), 2,4$-dimethoxybenzylamine $(225 \mu \mathrm{L}, 1.5 \mathrm{mmol})$ was added. The mixture was stirred for 30 minutes at room temperature. Then acid 2 was added $(297 \mathrm{mg}, 1.5$ $\mathrm{mmol})$ and the mixture was cooled to $0{ }^{\circ} \mathrm{C}$. Ethyl isocyanoacetate was added $(160 \mu \mathrm{L}, 1.5$ mmol). The reaction was continued for $24 \mathrm{~h}$. The solvent was evaporated and the product was purified by column chromatography (hexane: ethyl acetate, $6: 4 \mathrm{v}: \mathrm{v}) .401 \mathrm{mg}(0.73 \mathrm{mmol})$ of colorless oil was obtained (49\%). ${ }^{1} \mathrm{H}$ NMR (400 MHz, $\left.\mathrm{CDCl}_{3}\right): \delta 0.83-0,85\left(\mathrm{~m}, 6 \mathrm{H}, 2 \mathrm{x}-\mathrm{CH}_{3}\right)$, 1.20-1.34, (t, J $\left.7 \mathrm{~Hz}, 3 \mathrm{H}, \mathrm{OCH}_{2} \mathrm{CH}_{3}\right), 1.38-1.63\left(\mathrm{~m}, 10 \mathrm{H}, 5 \mathrm{x}-\left(\mathrm{CH}_{2}\right)-\right), 1.74-1.92(\mathrm{~m}$, $\left.1 \mathrm{H}, \mathrm{CH}\left(\mathrm{CH}_{3}\right)_{2}\right), 1.96-2.06\left(\mathrm{~m}, 2 \mathrm{H},-\mathrm{CH}_{2}-\right), 3.15-3.30\left(\mathrm{~m}, 1 \mathrm{H},-\mathrm{CH}\left(\mathrm{CH}_{2} \mathrm{O}-\right)_{2}\right), 3.78-4.00(\mathrm{~m}, 10 \mathrm{H}$, $\left.2 \mathrm{xOCH}_{3} ; 2 \mathrm{x}-\mathrm{CH}_{2}-\right)$, 4.12-4.34 (m, 4H, 2x- $\left.\mathrm{CH}_{2}-\right)$, 4.45-4.58 (s, 2H, $\left.\mathrm{CH}_{2}\right)$,4.78-4.91 (m, 1H, $\mathrm{CH}(i \mathrm{Bu})-), 6.37-6.50(\mathrm{~m}, 2 \mathrm{H}, 2 \mathrm{xArH}), 6.77-6.83(\mathrm{~m}, 1 \mathrm{H}, \mathrm{NH}), 6.85-7.00(\mathrm{~m}, 1 \mathrm{H}, \mathrm{ArH}) .{ }^{13} \mathrm{CNMR}$ $\left(100 \mathrm{MHz}, \mathrm{CDCl}_{3}\right): \delta 14.5,22.6,22.7,22.8,23.0,25.5,26.0,27.6,36.7,38.4,40.0,41.4,44.7$, 55.6, 56.3, 61.5, 61.6, 98.4, 98.8, 104.1, 117.5, 127.9, 158.0, 160.8, 169.8, 171.3, 173.6. Anal. calcd. for $\mathrm{C}_{29} \mathrm{H}_{44} \mathrm{~N}_{2} \mathrm{O}_{8}$ : C, 63.48; H, 8.08; N, 5.11; found: C, 63.52; H, 8.14; N, 4.95; HR-MS (ESI, $\left[\mathrm{M}+\mathrm{Na}^{+}\right]$) calculated for: $\mathrm{C}_{29} \mathrm{H}_{44} \mathrm{~N}_{2} \mathrm{O}_{8} \mathrm{Na}$ : 571.2990; found: 571.2995.

Synthesis of \{2-[(2,4-Dimethoxybenzyl)-(3-hydroxy-2-hydroxymethylpropionyl)-amino]-4methylpentanoylamino acetic acid ethyl ester (2). Compound 7 (400 mg, $0.73 \mathrm{mmol}$ ) was dissolved in $80 \%$ aqueous acetic acid $(6 \mathrm{~mL})$ and stirred for $24 \mathrm{~h}$ at room temperature. The solvent was evaporated and the product was purified by column chromatography (hexane : ethyl acetate, 6:4 v:v). Product (262 mg, $0.56 \mathrm{mmol})$ was isolated as white crystals $(77 \%)$. ${ }^{1} \mathrm{H} \mathrm{NMR}$ $\left(400 \mathrm{MHz}, \mathrm{CDCl}_{3}\right): \delta 0.76\left(\mathrm{~d}, J 6.4 \mathrm{~Hz}, 3 \mathrm{H},-\mathrm{CH}_{3}\right), 0.83\left(\mathrm{~d}, J 6.8 \mathrm{~Hz}, 3 \mathrm{H},-\mathrm{CH}_{3}\right) 1.23-1.33(\mathrm{t}, J$ $\left.7.2 \mathrm{~Hz}, 3 \mathrm{H},-\mathrm{CH}_{2} \mathrm{CH}_{3}\right), 1.34-1.38(\mathrm{~m}, 1 \mathrm{H}, \mathrm{CHH}(i \mathrm{Pr})), 1.43-1.55(\mathrm{~m}, 1 \mathrm{H}, \mathrm{CHH}(i \mathrm{Pr})), 1.83-1.97$ (m, 1H, $\left.\mathrm{CH}\left(\mathrm{CH}_{3}\right)_{2}\right), 3.07-3.15\left(\mathrm{~m}, 1 \mathrm{H}, \mathrm{CH}\left(\mathrm{CH}_{2} \mathrm{O}-\right)_{2}\right), 3.35-3.48(\mathrm{~m}, 2 \mathrm{H} 2 \mathrm{x}-\mathrm{OH})$ ), 3.70-3.88 (m, 10H, 2x-( $\left(\mathrm{CH}_{2}\right)-;$ 2x-OCH 3$), 4.05$ 0-4.20 (m, 4H, 2x-( $\left.\left.\mathrm{CH}_{2}\right)-\right), 4.45$ (d, J 6Hz, 1H, -CHH-), 4.76 (d, J 6.1 Hz, 1H, -CHH-) 4.90-4.99 (m, 1H, -CH(i-Bu)), 6.40-6.47 (m, 2H, ArH), 7-7.05 (m, 1H, $\mathrm{ArH}), 7.15-7.20$ (m, 1H, NH). ${ }^{13} \mathrm{C} \mathrm{NMR}\left(100 \mathrm{MHz}, \mathrm{CDCl}_{3}\right) \delta 14.4,22.7,23.0,25.8,36.6,41.4$, 44.9, 46.5, 55.5, 55.7, 56.8, 62.1, 62.4, 63.2, 98.8, 104.0, 117.7, 128.1, 157.9, 160.8, 171.2. HRMS (ESI, [M+Na ${ }^{+}$) calculated for: $\mathrm{C}_{23} \mathrm{H}_{36} \mathrm{~N}_{2} \mathrm{O}_{8} \mathrm{Na}$ 491.2364; found: 491.2375. HPLC: Method $\mathrm{B}: t_{\mathrm{R} 1}=17.4 \mathrm{~min}, t_{\mathrm{R} 2}=19.8 \mathrm{~min}$; Method $\mathrm{C}: t_{\mathrm{R}}=2.6 \mathrm{~min}$.

\section{\{2-[(3-Acetoxy-2-hydroxymethylpropionyl)benzylamino]-4-methylpentanoylamino\}acetic}

acid benzyl ester (4). Chemical synthesis. A solution of 1 (68 $\mathrm{mg}, 0.14 \mathrm{mmol}$ ) and triethylamine $(49 \mu \mathrm{L}, 0.35 \mathrm{mmol})$ and DMAP $(10 \mathrm{mg})$ in dry methylene chloride $(2 \mathrm{~mL})$ was cooled to $0{ }^{\circ} \mathrm{C}$. Acetyl chloride was added dropwise $(21 \mu \mathrm{L}, 0.29 \mathrm{mmol})$ at $0{ }^{\circ} \mathrm{C}$. The reaction was carried out at room temperature for $18 \mathrm{~h}$. Water $(5 \mathrm{~mL})$ was added and the mixture was washed with sodium carbonate $(10 \%, 5 \mathrm{~mL})$. The organic layer was dried over magnesium sulphate and the solvent was evaporated. The monoacylated compound (35 mg) was isolated as a mixture of diastereoisomers using flash chromatography (silica gel, hexane:ethyl acetate) with $44 \%$ yield. Also diacylated compound was isolated as a byproduct (19 mg, 26\%). ${ }^{1} \mathrm{H}$ NMR $(200$ $\mathrm{MHz}, \mathrm{CDCl}_{3}$, mixture of diasteroisomers): $\delta 0.74(\mathrm{~d}, J 6.4 \mathrm{~Hz}, 3 \mathrm{H}), 0.84(\mathrm{~d}, J 6.4 \mathrm{~Hz}, 3 \mathrm{H}), 1.04-$ $1.58(\mathrm{~m}, 3 \mathrm{H}), 1.78-1.95(\mathrm{~m}, 1 \mathrm{H}), 2.00-2.10(\mathrm{~m}, 4 \mathrm{H}), 3.10-3.38(\mathrm{~m}, 1 \mathrm{H}), 3.64-4.48(\mathrm{~m}, 6 \mathrm{H}), 5.08-$ $5.38(\mathrm{~m}, 3 \mathrm{H}), 7.18-7.38(\mathrm{~m}, 12 \mathrm{H}) .{ }^{13} \mathrm{C} \mathrm{NMR}\left(50 \mathrm{MHz}, \mathrm{CDCl}_{3}\right): \delta 21.1,22.6,23.0,25.6,36.4$, 
41.3, 45.2, 47.9, 55.9, 62.8, 67.9, 126.3, 127.6, 128.8, 128.9, 129.0, 137.8, 171.3; HR-MS (ESI, $[\mathrm{M}+\mathrm{Na}]^{+}$) calculated for: $\mathrm{C}_{28} \mathrm{H}_{36} \mathrm{~N}_{2} \mathrm{O}_{7} \mathrm{Na}: 535.241$; found: 535.239 ; HPLC: Method A: $t_{\mathrm{R} 1}=$ $12.4 \mathrm{~min}, t_{\mathrm{R} 2}=13.4 \mathrm{~min}$

\{2-[(2,4-Dimethoxybenzyl)-(3-hydroxy-2-hydroxymethylpropionyl)amino]-4-methylpentanoylamino\}acetic acid. (5) as a reference compound. Into a solution of compound $2(54 \mathrm{mg}$, $0.12 \mathrm{mmol})$ in methanol $(2 \mathrm{~mL}), 1 \mathrm{~N} \mathrm{NaOH}(220 \mu \mathrm{L})$ was added. The mixture was stirred for $1 \mathrm{~h}$ at room temperature. Solvent was evaporated and crude product was analyzed by HR-MS without purification. HR-MS (ESI, [M- $\left.\mathrm{H}^{+}\right]$) calculated for $\mathrm{C}_{21} \mathrm{H}_{31} \mathrm{~N}_{2} \mathrm{O}_{8}$ : 439.2075, found 439.2075.

Enzymatic reactions. All enzymes apart acetone powders and Novosym 435 were purchased from Sigma-Aldrich. Initial screenings were performed with the following enzymes: Novosym 435, amano lipase PS, papaine, lipase from wheat germ, acylase I, amano lipase AK, lipase from hog pancreas, Porcine pancreas lipase type II, Macerase Pektynase from Rhizopus $s p$. as well as recombined lipases from: Rhisopus niveus, Rhisopus oryzae, Rhisopus arrhisus, Candida lipolitica, Mucor javanicus, Penicilium roqeufori, Candida antarctica, Pseudomonas fluorescens, Pseudomonas cepaciae, Aspergillus oryzae. Also, the following acetone powders were applied: PLAP, GLAP, BLAP, RLAP, DLAP.

General method for enzymatic acylation. Compound 1 was dissolved in solvent (Table $1, \mathrm{c}=$ $10 \mathrm{mg} / \mathrm{mL}$ ) and enzyme was added (Table 1, $2 \mathrm{mg}$ for each $10 \mathrm{mg}$ of substrate). Vinyl acetate (3 eq) was added. The reaction was carried out at $40{ }^{\circ} \mathrm{C}$. Solvent was evaporated and the product was analyzed by HPLC (Method A).

General method for enzymatic hydrolysis. Compound $\mathbf{2}$ was dissolved in buffered 7.0/acetone $(8: 2, \mathrm{v}: \mathrm{v})$ and the enzyme was added (Table $2,10 \% \mathrm{~g})$. The reaction was carried out at room temperature $\left(25^{\circ} \mathrm{C}\right)$. Acetone was then evaporated and aqueous phase was extracted with ethyl acetate. The organic layers was combined and washed with $1 \mathrm{~N} \mathrm{NaHCO}_{3}(3 \mathrm{x})$. The ethyl acetate was evaporated. The product was analyzed by HPLC (Method B).

\section{Acknowledgements}

This work was supported by project "Biotransformations for pharmaceutical and cosmetics industry" No. POIG.01.03.01-00-158/09-01 part-financed by the European Union within the European Regional Development Fund. 


\section{References}

1. Bornscheuer, U. T.; Kazlauskas, R. J. Hydrolases In Organic Synthesis; Wiley-VCH: New York, 1999.

2. Faber, K.; Faber, K. Biotransformations in Organic Chemistry; SpringerVerlag: Berlin, 2004. http://dx.doi.org/10.1007/978-3-642-18537-3

3. Drauz, K.; Groger, H.; May, O. Enzyme Catalysis in Organic Synthesis; WileyVCH:Weinheim, 2013. PMid:23339093

4. Szymanski, W.; Ostaszewski, R. Tetrahedron 2008, 64, 3197. http://dx.doi.org/10.1016/j.tet.2008.01.103

5. Fryszkowska, A.; Frelek, J.; Ostaszewski, R. Tetrahedron 2005, 61, 6064. http://dx.doi.org/10.1016/j.tet.2005.04.032

6. Znabet, A.; Ruijter, E.; Kanter, F. J. J. de, Kohler, V.; Helliwell, M.; Turner, N. J.; Orru, R. V. A. Angew. Chem. Int. Ed. 2010, 49, 5289.

http://dx.doi.org/10.1002/anie.201001592

PMid:20575127

7. Dommling, A.; Ugi, I. Angew. Chem. Int. Ed. 2000, 39, 3168. http://dx.doi.org/10.1002/1521-3773(20000915)39:18<3168::AID-ANIE3168>3.0.CO;2-U

8. Zhu, J.; Bienayme, H. Multicomponent Reactions; Wiley-VCH:Weinheim, 2005. http://dx.doi.org/10.1002/3527605118

9. Dömling, A.; Wang, W.; Wang, K. Chem. Rev. 2012, 112, 3083. http://dx.doi.org/10.1021/cr100233r PMid:22435608

10. Ramon, D. J.; Yus, M. Angew. Chem. Int. Ed. 2005, 44, 1602. http://dx.doi.org/10.1002/anie.200460548 PMid:15719349

11. Yue, T.; Wang, J.; Wang, D.; Masson, G.; Zhu, J. Angew. Chem. Int. Ed. 2009, 48, 6717 http://dx.doi.org/10.1002/anie.200902385 PMid:19658144

12. Su, Y.; Bouma, M. J.; Alcaraz, L.; Stocks, M.; Furber, M.; Masson, G.; Zhu, J. Chem. Eur. J. 2012, 18, 12624

http://dx.doi.org/10.1002/chem.201202174

PMid:22930594

13. Hashimoto, T.; Kimura, H.; Kawamata, Y.; Maruoka, K. Angew. Chem. Int. Ed. 2012, 51, 7279

http://dx.doi.org/10.1002/anie.201201905

PMid:22692817 
14. Parkkari, T.; Savinainen, J. R.; Raitio, K. H.; Saario, S. M.; Matilainen, L.; Sirvioe, T.;

Laitinen, J. T.; Nevalainen, T.; Niemi, R.; Jaervinen, T. Bioorg. Med. Chem. 2006, 14, 5252. http://dx.doi.org/10.1016/j.bmc.2006.03.051

PMid:16644227

15. Scriba, G. K. E.; Lambert, D. M. Pharm. Res. 1997, 14, 251.

http://dx.doi.org/10.1023/A:1012073332415

PMid:9090719

16. Gluchowski, C.; Bischoff, T. E.; Garst, M. E.; Kaplan, L. J.; Dietrich, S. W.; Aswad, A. S.; Gaffney, M. A.; Aoki, R.; Garcia, C.; Wheeler, L. A. J. Med. Chem. 1991, 34, 392. http://dx.doi.org/10.1021/jm00105a060

17. Klossowski, S.; Muchowicz, A.; Firczuk, M.; Redzej, A.; Swiech, M.; Golab, J.; Ostaszewski, R. J. Med. Chem. 2012, 55, 55.

http://dx.doi.org/10.1021/jm201359d

PMid:22128876

18. Noryoshi, A.; Chikaraishi, N.; Ikawa, M.; Omura, S.; Kuwajima, I. Tetrahedron: Assymmetry 2004, 15, 733.

http://dx.doi.org/10.1016/j.tetasy.2004.01.007

19. Szymanski, W.; Ostaszewski, R. Tetrahedron: Asymmetry 2006, 17, 2667.

http://dx.doi.org/10.1016/j.tetasy.2006.09.014 